\title{
Partial View Modeling and Validation in 3D Laser Scans for Grasping
}

\author{
Nico Blodow, Radu Bogdan Rusu, Zoltan Csaba Marton, Michael Beetz \\ Intelligent Autonomous Systems, Technische Universität München \\ Boltzmannstr. 3, Garching bei München, 85748, Germany \\ $\{$ blodow, rusu, marton, beetz\}@cs.tum. edu
}

\begin{abstract}
Humanoid robots performing every day tasks in human environments need a strong perception system in order to operate successfully. As 3D data acquisition devices like laser scanners and time of flight cameras get better and cheaper, we expect three-dimensional perception to become more important. We describe a new method for detecting surfaces of revolution in point clouds within our Sample Consensus Framework. Cylinders, cones and arbitrary rotational surfaces can be reliably and efficiently detected. Symmetry assumptions can be hypothesized and verified in order to complete the model from a single view, i.e. to generate data on the occluded parts of the object. These complete models can be used for grasp analysis. Additionally, we propose a new method for scoring models within the Sample Consensus Framework in order to get better shapes.
\end{abstract}

\section{INTRODUCTION}

Two of the key challenges for humanoid robots performing useful tasks in human environments is manipulation of objects, which in turn needs a perception system that enables the robot to operate successfully. Constructing a 3D map to prevent collisions while navigating and moving and detection of objects of interest for successful manipulation are very important abilities the perception system needs to have.

In our scenario, we are working at enabling a mobile robot platform to perform useful tasks like cleaning, setting the table and manipulating objects within a human household setting. We are striving for robust and efficient detection of obstacles, tables and furniture, doors, and objects that can be manipulated. In previous work, we have applied a wide range of Sample Consensus based methods with different underlying model types for detection of planes, cylinders, cones, and spheres. We combined these methods with a top-down segmentation and reasoning approach in order to reduce the search space and to be able to semantically label detected shapes and objects like kitchen furniture, appliances, walls, floor and ceiling [1].

However, geometric shapes as the ones mentioned above, while very common in human environments, are in general not sufficient to model everything the robot might encounter, such as china cups, vases and other surfaces of revolution. Therefore, we developed a novel model that can capture these rotationally symmetric shapes in unstructured point clouds.

One interesting property of this class of objects is that they have rotational symmetry. That means, from a partial view of the surface, we can generate a hypothetic complete model, which we actually use for two reasons: for testing the plausibility of a hypothetic model, that is, to decide whether there are parts of the hypothesized surface that are contradicted by the measured data. And, secondly, for completion of objects from partial views. This is important as we want to use the detected geometry to perform manipulation, and common grasp planners like GraspIt! [2] and OpenRAVE [3] rely on complete models to compute feasible grasps.

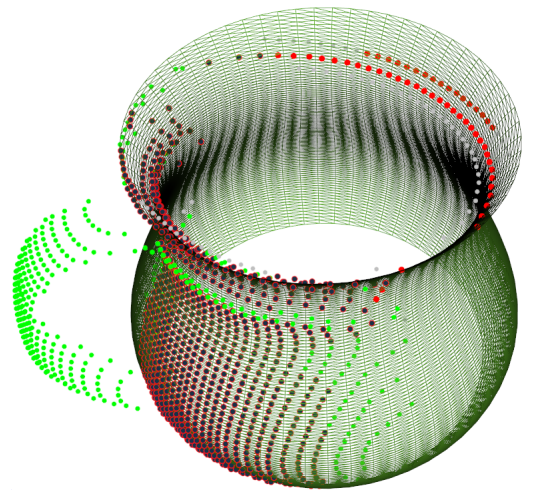

Fig. 1. A surface of revolution reconstructed from a partial view. The supporting inlier points are marked in red, and green points represent possible handle points.

It is also possible to detect handles in a scene [4], which is very useful since handles are made to be manipulable by humans, so they are a good starting points for humanoids. It is on the other hand limited to objects that have a handle that is also visible from the current view point.

\section{RELATED WORKS}

object or shape reconstruction has been widely studied in literature. Among the most important are the Hough transform [5], [6] and RANSAC [7]. The former, however, becomes inefficient as the dimensionality of the parameter space increases. RANSAC was originally applied to detect cylinders from range data, and numerous improvements and alterations of the basic approach have been proposed since its introduction. Some methods change the way the different models are scored and compared against each other, for example MSAC [8], which weighs points by their distance to the model. However, the authors know of no RANSAC based method that can extract arbitrary surfaces of revolution (see Figure 1).

Some, if not all of these methods have been developed to solve the stereo vision problem of estimating the epipolar geometry from sets of correspondences of two or more 
images. However, we are applying these general methods on scanned data. In 2.5 dimensional scans like ours, each point of the point cloud also tells that the space in the line of sight between the scanner and the point is not occluded. This means we can label space with four tags: occupied, where there are surface points detected by the scanner, free, where the laser ray has passed through without hitting an object, occluded, which marks regions in space that are behind the object from the laser scanner's point of view, and unknown, the space that was not covered by a view frustrum. Note that this applies to general 3D scans as well as long as for every point in the cloud, the point it has been seen from (the viewpoint) is known.

Similar methods have been used in the viewpoint planning domain and collision avoidance, for example in [9], where the workspace has been voxelized and labeled with similar tags. Due to the spatial dimensions of these voxels however the labeling is not completely correct, since a voxel can contain a combination of these "space types". Increasing the resolution too much and covering every possible scenario is not feasable, but we found that it is sufficient if we make the following specifications:

- because the laser beams don't cover all of the space continously, technically there will allways be unknown space between laser rays, but for simplicity, we consider any information that we gain about a voxel to overwrite the unknown label.

- the occupied label encompasses also the free and occluded ones, since the voxel contains free space in front of the measurement point, occupied space at the point, and occluded space behind it.

- a voxel can have both the free and occluded labels if only parts of it are occluded by points in the foreground.

This labeled voxel representation will be used in section IVA to assess the plausibility of hypothetical model candidates.

Often in 3D Sample Consensus based methods that use one of the classic model scoring functions, false positives are generated in the process that get a lot of support (for example inliers) even though the models are clearly not good for some reason. For example, disconnected regions in space belonging to the same plane, or noisy parts of the scene that get picked up as a sphere, or corners of a box that look like a cylinder to the algorithm.

There have been a number of remedies to fix these issues, like making sure that the final inlier set is the biggest connected cluster of the initial inliers [10]. This is of course computationally expensive, and depending on the applied algorithm, is sensitive to the connectivity bitmap grid resolution or the distance threshold used to seperate clusters of points.

Another step that is useful and easily doable in the presence of surface normals are normal checks per inlier, i.e. if a point is inlier based on the distance to the model, one can additionally ensure that the corresponding (estimated) surface normal is in accordance with the underlying model's surface normal. This is however hard to incorporate in a scoring function other than the basic inlier counting method.
We therefore propose a new scoring function tailored to the application of detecting shapes in $2.5 \mathrm{D}$ depth images. We think it is important to assess the quality of a hypothesized model by looking at a) its support (how do the present points support the model) and b) the lack of contrary evidence (do parts of the model lie within free space).

The key contributions of the research presented in this paper are a) a novel Sample Consensus based method exploiting knowledge about occluded and empty space to find better models, and b) a novel Sample Consensus model that allows detection of surfaces of revolution, including, but not limited to cylinders, cones and sphere. This will later be used to perform partial view manipulation.

The remainder of this paper is organized as follows: In section III, we will give a overview of our system architectur, followed by IV, where we will present our proposed addition to the RANSAC algorithm. Then, in V, we will describe our proposed method of detecting surfaces of revolution, and explain the advantages that both these ideas have in the context of perception for manipulation in chapter VI-B. We will show experimental results in VI and outline our intended future research in section VII

\section{SYSTEM OVERVIEW}

We approach the problem of detecting objects for manipulation by combining some heuristics about the structure of human environments with a smart selection of processing steps to achieve effective and efficient results. Our Semantic Mapping pipeline is described in detail in [1], [4], however the basics are outlined as follows.

We start by acquiring 3D point cloud data by either sweeping a 2D laser scanner mounted on the robot arm or from a time of flight camera. This data is slightly processed to remove sparse outliers and to remove some noisy measurements. Also, we estimate surface normals by performing Principal Component Analysis on local point neighborhoods.

Since we assume that the world up axis is known in these data sets, we can quickly detect table surfaces using common sense rules about their geometric extents and height, using the subset of the point cloud that have vertical surface normals. We then create bounding polygons for these planar table surfaces, and cluster the points on top of the tables into groups based on a connectivity criterion in a downsampled octree representation of the scene. This leaves us with seperate disjoint clusters, which greatly speeds up detection, as most points from a cluster will with a high probability belong to the same object.

Parallel to this, we also compute the voxelized grid representation of our scene that holds information about occlusions and empty regions in space. We use raycasting from the viewpoint(s) to each point measurement to perform this labeling. Note that not all voxels have to be tested for intersection with the ray if the ray is simply traversed voxel by voxel starting at the viewpoint, allowing for much faster processing.

Note that in our implementation, we employed a nonhierarchical voxelization. This can lead to significant memory re- 
quirements, depending on the desired grid resolution. While we did not experience this in our experiments, constructing the grid only in the vicinity of the table, this could be optimized using a octree or similar spatial decomposition. This would decrease memory consumption at the expense of lookup speed (logarithmic instead of constant).

\section{SAMPLE CONSENSUS METHOD}

The basic concept of the Sample Consensus Methods as first proposed by [7] is straightforward yet powerful: In principle, the following steps are repeated:

1) SAMPLING - select random $m$-subset of input points

2) MODEL ESTIMATION - compute model parameters

3) MODEL VERIFICATION - evaluate scoring function

4) MODEL EXTRACTION - accept best model

Assume a point cloud $\mathcal{P}$, with $\|\mathcal{P}\|=N$ points $\left(\boldsymbol{p}_{1} \cdots \boldsymbol{p}_{N}\right)$. Iteratively, random $m$-subsets of this input data set are selected and parameters of a model $\mathcal{M}$ fitting the sample points in this subset are computed. The term model will be used both for the set of sample points points and the parameters, depending on the context. The model gets scored using a scoring scheme, which in its basic form can be the cardinality of the set of inliers, i.e. the input data points $\boldsymbol{p}_{i}$ whose distance $d\left(\boldsymbol{p}_{i}, \mathcal{M}\right)$ to the model was smaller than a certain threshold $d_{t h}$. In the last step, the current best model gets extracted if the probablity $p(\mathcal{M})$ is high enough that the algorithm will fail to find a better model.

In each of these steps, we strive to tune the algorithm to optimize performance, both computational wise and to improve the quality of the extracted models. In detail, these improvements are: in the Sampling step, we decrease the expected number of iterations by utilizing a octree for spatial decomposition, which allows us to use a localized sampling strategy, which increases the chances that the sampled points will lead to a decent model. This has been described in [10]. In summary, the probability $p(\mathcal{M})$ improves from the original RANSAC form of

$$
P(\mathcal{M}) \approx\left(\frac{\|\mathcal{M}\|}{N}\right)^{m}
$$

to

$$
P_{\text {local }}(\mathcal{M}) \approx \frac{\|\mathcal{M}\|}{N l 2^{m-1}},
$$

where $l$ denotes the depth of the octree. With $l=7$, the improvement factor ranged from 10 to 350 in our experiments.

The Model Estimation step obviously depends heavily on the kind of shape that should be detected. In our case, we are interested in rotational objects, which are common in household environments and also facilitate $3 \mathrm{D}$ reconstruction from partial views. This step is presented in detail in section V.

In the Model Verification stage, the scoring function is used to establish a partial ordering of the different model hypothesis. In its simplest form, counting the inliers corresponds to the following value function $v$ that should be maximal for a given shape model $\mathcal{M}$ :

$$
\begin{aligned}
v_{(\mathcal{M})} & =\sum_{i \in N} \varepsilon_{\left(\mathcal{M}, \boldsymbol{p}_{i}\right)} \\
\varepsilon_{(\mathcal{M}, \boldsymbol{p})} & = \begin{cases}1, & \text { if } d\left(\boldsymbol{p}, \mathcal{M}_{k}\right)<d_{t h} \\
0, & \text { else }\end{cases}
\end{aligned}
$$

\section{A. Evidence-based Scoring Scheme}

However, the points in the point cloud do not only provide information about the space immediately around their position, but also about the space between the point and the origin of the laser ray. Therefore it makes sense to incorporate information about contradicting evidence into the score for a model.

Specifically, we propose the following addition to Eq. 3: We introduce a plausibility score $s$ for a model, and use it to scale $v_{(\mathcal{M})}$.

$$
v_{(\mathcal{M})}=s \cdot \sum_{i \in N} \varepsilon_{\left(\mathcal{M}, \boldsymbol{p}_{i}\right)}
$$

Special care has to be taken to ensure that this score does not act arbitrarily on the relative quality of a model compared to another one. Otherwise, it is not clear whether a large object with low plausibility should be valued above a small, but very plausible model.

To compute a score for a given model, we perform the following steps:

1) For a given model $\mathcal{M}$, we generate a mesh of the complete objects as explained in $\mathrm{V}$.

2) We then construct a kd-tree of the mesh vertices for fast neighborhood searches.

3) For every inlier point $\boldsymbol{p}_{q}$ to $\mathcal{M}$, we select all mesh vertices that are within a certain radius of the query point $\boldsymbol{p}_{q}$ and mark those vertices as seen vertices. Let their number be $v_{s}$.

4) For the unmarked rest of the vertices, we query the voxel representation of the free space to see whether that part of the surface would come to lie in space that the laser scanner found to be free of any objects. Vertices that are in occluded space get marked as occluded vertices. Let their number be $v_{o}$.

5) The final score $s$ for the model is then defined as:

$$
s=\frac{v_{o}+v_{s}}{v_{s}}
$$

This way, multiplying the number of inliers with this score amounts to counting also the possible unseen points in occluded space.

\section{SURFACES OF REVOLUTION}

A surface of revolution is generated by rotating a $2 \mathrm{D}$ curve around an axis. Detecting such a shape in a 3D laser scan requires an estimate for the axis as well as for the curve. For our experiments, we decided to use polynomial curves because approximating points with bezier splines is computationally too expensive since it requires an iterative approximation algorithm (for example [11]) in every RANSAC iteration and for every model hypothesis. 


\section{A. Estimating Shape Parameters from Sample Points}

We tackled the problem of determining suitable shape parameters in two steps, by first estimating the axis from the sample points and their normals, and then computing the approximating polynome's coefficients. This is done by rotating a $2 \mathrm{D}$ plane (containing the axis) around the axis and collecting the sample points in it. We then perform a polynomial interpolation through these $2 \mathrm{D}$ points as an initial guess for a curve.

This means we need to sample $m$-subsets of $\mathcal{P}$ with $m=$ $n+1$, where $n$ is the order of the polynomial. Given these $m$ points, it is no clear how to analytically compute an axis. However, if the estimated normals are closely similar to the correct surface normals, the line-to-line distance between the axis and each line defined by a sample point and its normal should be close to zero. Let $\langle\boldsymbol{a}, \overrightarrow{\boldsymbol{a}}\rangle$ denote the axis, defined by a point $\boldsymbol{a}$ and a direction vector $\overrightarrow{\boldsymbol{a}}$ and let $\left\langle\boldsymbol{p}_{i}, \overrightarrow{\boldsymbol{n}}_{i}\right\rangle$ denote the $i$ th sample point and its corresponding normal vector. Then, we minimize the following function over $\boldsymbol{a}$ and $\overrightarrow{\boldsymbol{a}}$ :

$$
\sum_{i=0}^{m} d_{l, l}\left(\langle\boldsymbol{a}, \overrightarrow{\boldsymbol{a}}\rangle,\left\langle\boldsymbol{p}_{i}, \overrightarrow{\boldsymbol{n}}_{i}\right\rangle\right)^{2},
$$

where $d_{l, l}$ stands for the line-to-line distance.

The function minimization is done using a LevenbergMarquardt non-linear optimizer. As a starting solution, we take $\boldsymbol{a}$ to be the centroid of the sample points $\boldsymbol{a}:=$ $\frac{1}{m} \sum_{i=0}^{m} \boldsymbol{p}_{i}$, and the axis as the $z$-axis, i.e. pointing upwards. A lot of objects like cups, pots and so on commonly found in kitchens are close to upright anyway, but we were able to successfully detect objects with a horizontal axis just as easily, as the optimization step rotated the axis into the correct direction.

Once an axis estimate is available, one can iterate over the point cloud and project each point $\boldsymbol{p}_{i}$ onto the line $\langle\boldsymbol{a}, \overrightarrow{\boldsymbol{a}}\rangle$. Let the projected point be $\boldsymbol{q}_{i}$. Consider $\boldsymbol{a}_{0}$ to be the projection of the origin of the coordinate system onto the line. Then the 2D $x$-coordinate of each point can be taken as $d_{p, p}\left(\boldsymbol{p}_{i}, \boldsymbol{a}_{0}\right)=\left\|\boldsymbol{q}_{i}-\boldsymbol{a}_{0}\right\|$, and the 2D $y$-coordinate is the point-to-line distance $d_{p, l}\left(\boldsymbol{p}_{i},\langle\boldsymbol{a}, \overrightarrow{\boldsymbol{a}}\rangle\right)$. These $\langle x, y\rangle$ points can then be used to compute the coefficients $c_{j}, j \in\{0 \cdots n\}$ of the polynomial using well-known techniques for polynomial regression.

\section{B. Refitting the Shape Parameters to the Inlier Points}

As shown in [12], it is beneficial for the quality of the detected shape to perform a refitting step before extracting the final model. In our approach, we performed this step for the axis and the polynomial seperately. This means that given the current inliers, we performed the same optimization step as in the initial shape parameter estimation V-A. This is followed by a inlier search using the new axis. Finally, the polynomial coefficients get re-computed, given the new axis.

\section{EXPERIMENTAL RESULTS}

\section{A. Experiments and Results}

Experiments have been carried out to ensure the effective performance of the proposed solutions. in Figure 2, you can see a sample input data set as scanned from our acquisition system. One can see some spurious measurements, which can be filtered away easily. The objects are all surfaces of revolution, to demonstrate the large degree of variation our algorithm can pick up on. In Fig. 3, you can see the result of the online clustering algorithm. Different clusters are high lighted in different colors. Note how the pan in the far right corner of the table got segmented into two seperate clusters.

In Fig. 4 to 6, we show the results of the proposed system. The colors range from red to black, depending on the plausibility score. That means, the darker a shape is colored, the better the system thinks it is. For example, the oversegmented pan is very bright in all 3 images, since most parts of the rotational surface are not explained by points. In Fig.4, the score has not been used to find better shapes, only the basic RANSAC method has been used to show the feasibility of the rotational model, even without the additional score function. However, the shape of the pan on the far right corner is not correct. Also, the bowl on the far left corner got misdetected as a bigger object that lies a little on its side. However, since the parts of the surface that stick out over the actual object is in evidently empty space, our scoring function alleviates this problem, as can be seen in Fig. 5. Fig. 6 shows a similar result, however the point display size of the generated mesh vertices is smaller.

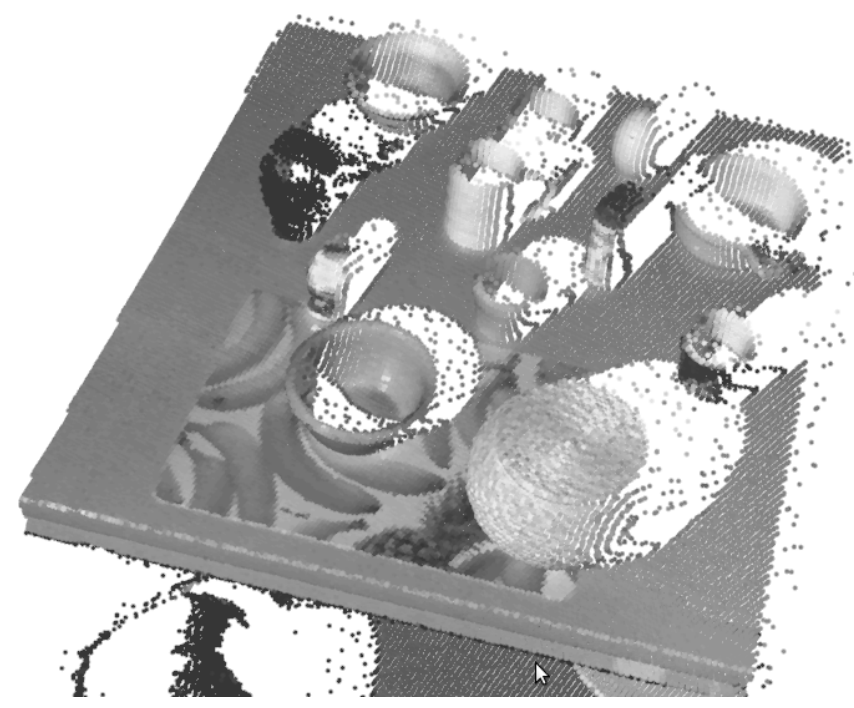

Fig. 2. Original input data. Point brightness reflects intensity information from the scanner, which is dense enough to capture the texture of the placemat

\section{B. Partial View Grasping}

Preliminary experiments have been conducted in simulation regarding the planning and execution of grasping motions for objects that have only partially be observed. Common grasp-planning software systems rely on complete models from a complete $360^{\circ}$-scan of an object or from a $\mathrm{CAD}$ model. If the perception system sees an object for the first time and does not have a complete scan, these system will fail to find a feasible and successful grasp. 


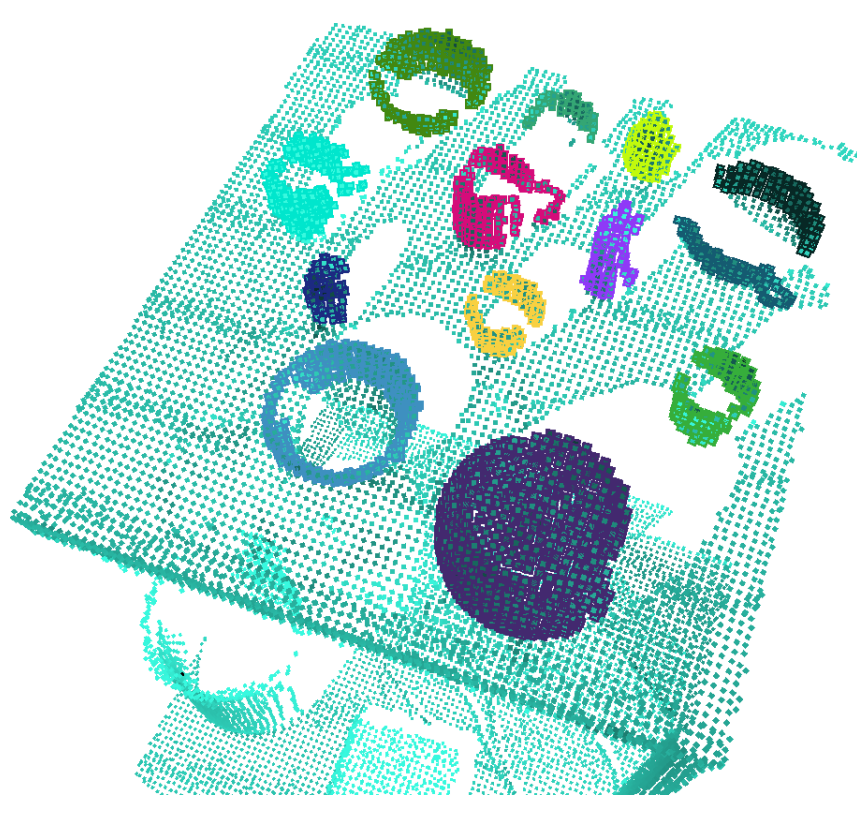

Fig. 3. Clusterization of the objects on table surfaces.

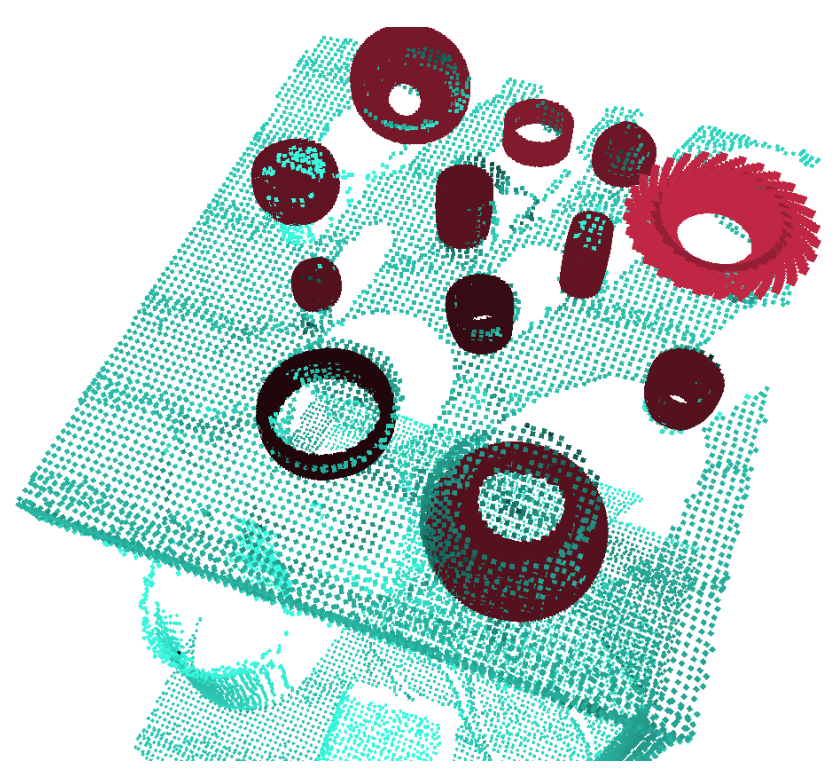

Fig. 4. Results for the Classical RANSAC approach.

In Figure 1, you can see a surface of revolution that has been reconstructed from a partial view of a cup. Red points show the inliers after the final refitting step, and points on handles are marked in green. Handle points were found using a simple heuristic: they are points that are connected to the surface of the object, and were therefore grown from the inliers (see [4] for details). Incorporating the knowledge about empty and occluded space allows the object to be completed. The resulting mesh can be used for grasp planning, as shown in Figure VI-A. It shows a grasping experiment, where the located and reconstructed objects are grasped by our kitchen manipulation platform. Note that for this experiment, no additional sensors have been employed

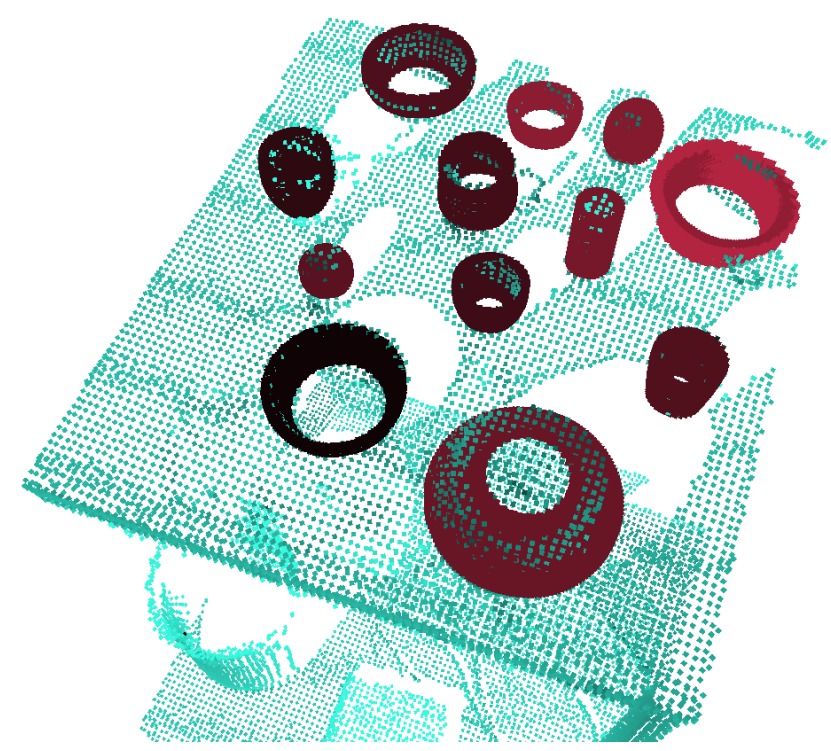

Fig. 5. Resulting objects for our RANSAC method using a plausibility score based on knowledge about occlusions and empty space.

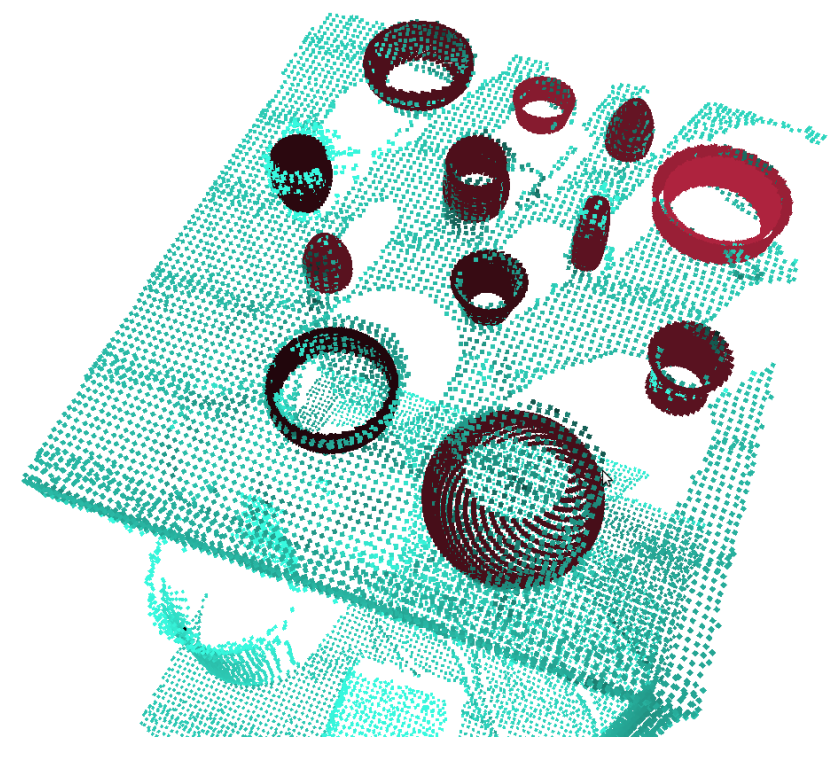

Fig. 6. Similar to Fig. 5. Shown to demonstrate the random nature of the algorithm.

than the tilting 2D laser scanner on the robot's shoulder.

\section{CONCLUSIONS AND FUTURE WORKS}

\section{A. Conclusions}

We have presented a method for generating complete 3D models of objects with rotational symmetry from partial views and their potential use for grasping. We desribed a method that allows exploiting intrinsic knowledge in a $2.5 \mathrm{D}$ laser scan about free space in order to help finding better shapes. We specifically presented a novel approach for the detection of surfaces of revolution that works in unstructured point clouds as well as in $2.5 \mathrm{D}$ scans. It was shown how the system can infer missing knowledge about hidden and 

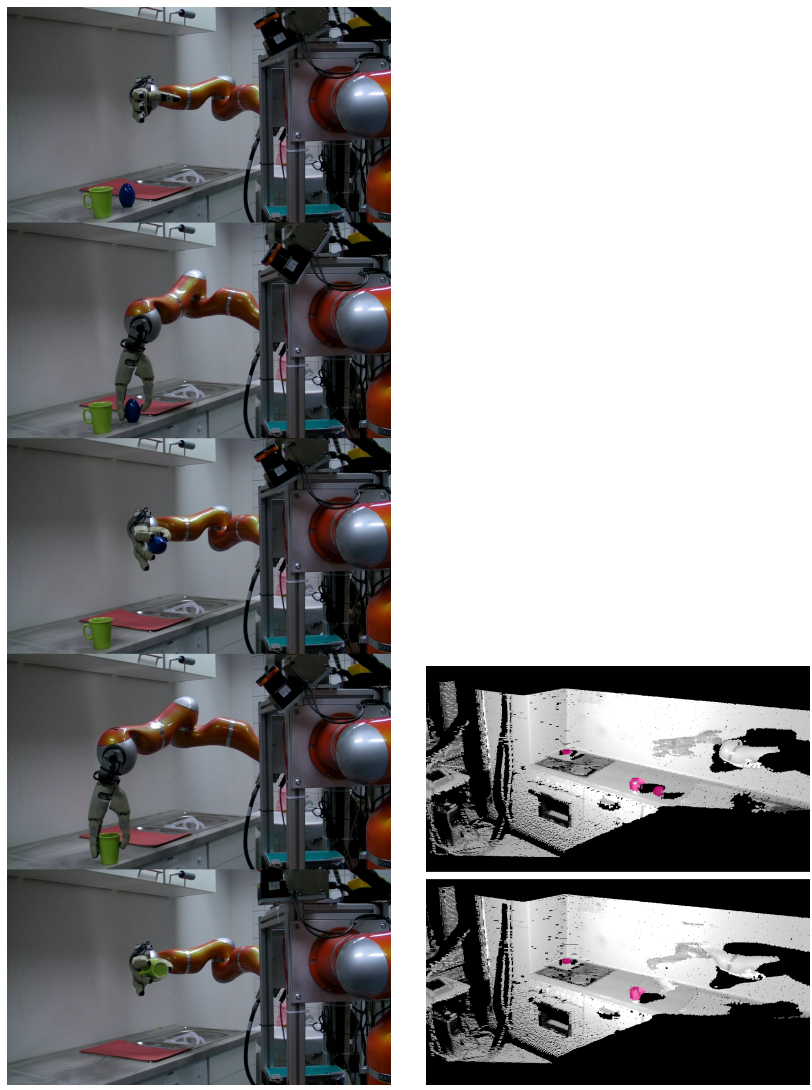

(a) Still images from autonomous (b) Corresponding point clouds acgrasping experiment

quired between arm movements

Fig. 7. A successful autonomous grasp experiment in our kitchen scenario

occluded surfaces by validating rotational symmetry assumptions.

\section{B. Future Works}

In the future, we will make more shape models aware of the implications of symmetry and occluded/empty space. A particularly interesting shape is the box, as it is very common and usually detected as single planes. However, exploiting the inherent planar symmetries of boxes, together with their plausibility in a scan given the score method presented here will likely lead to a more symbolic formulation of the concept "box". Grasping is not too far-fetched from there, as the graspability of the box can easily be verified by checking if the lengths of the edges are bigger than what the robot hand can safely grip.

Since the system presented here was developed with the goal in mind that it shall be employed within a real personal robotics scenario, it is clear that more experiments have to be performed in the future, with different models and different amounts of clutter.
Also, a low plausibility score can also lead to the realization that the data present in the current scan might not be enough to reliably detect objects. In this case, the system might decide that additional scans must be taken, and the placement of the sensor for the next best view can be facilitated by having a hypothesized back side available. Automatic sensor path or pose planning might benefit especially from the fact that backsides of objects with a very high score don't need to be scanned on the back, therefore the system could decide which data acquisition action might be most suitable at a given point in time.

\section{ACKNOWLEDGMENTS}

This work has been funded by the Cognition for Technical Systems (CoTeSys) Cluster of Excellence.

\section{REFERENCES}

[1] R. B. Rusu, Z. C. Marton, N. Blodow, M. Dolha, and M. Beetz, "Towards 3D Point Cloud Based Object Maps for Household Environments," Robotics and Autonomous Systems Journal (Special Issue on Semantic Knowledge), 2008.

[2] A. Miller and P. K. Allen, "Graspit!: A Versatile Simulator for Robotic Grasping," IEEE Robotics and Automation Magazine, vol. 11, no. 4, pp. 110-122, 2004.

[3] R. Diankov and J. Kuffner, "Openrave: A planning architecture for autonomous robotics," Robotics Institute, Pittsburgh, PA, Tech. Rep. CMU-RI-TR-08-34, July 2008.

[4] R. B. Rusu, N. Blodow, Z. C. Marton, and M. Beetz, "Close-range Scene Segmentation and Reconstruction of 3D Point Cloud Maps for Mobile Manipulation in Human Environments," in Proceedings of the IEEE/RSJ International Conference on Intelligent Robots and Systems (IROS), St. Louis, MO, USA, October 11-15 2009.

[5] T. Rabbani and F. Heuvel, "Efficient Hough Transform for Automatic Detection of Cylinder in Point Clouds," in ISPRS WG III/3, III/4, V/3 Workshop, Laser scanning 2005, Enschede, The Netherlands, 2005.

[6] G. Vosselman and S. Dijkman, "3d building model reconstruction from point clouds and ground plans," in International Archives of Photogrammetry and Remote Sensing, Volume XXXIV-3/W4 pages 3743,Annapolis, MD, 22-24 Oct. 2001, 2005.

[7] M. A. Fischler and R. C. Bolles, "Random Sample Consensus: A Paradigm for Model Fitting with Applications to Image Analysis and Automated Cartography," in Comm. of the ACM, Vol 24, 1981, 1981.

[8] P. Torr and A. Zisserman, "MLESAC: A new robust estimator with application to estimating image geometry," Computer Vision and Image Understanding, vol. 78, pp. 138-156, 2000.

[9] P. Renton, M. Greenspan, H. A. Elmaraghy, and H. Zghal, "Plan-nscan: A robotic system for collision-free autonomous exploration and workspace mapping," J. Intell. Robotics Syst., vol. 24, no. 3, pp. $207-$ 234, 1999.

[10] R. Schnabel, R. Wahl, and R. Klein, "Efficient RANSAC for PointCloud Shape Detection," Computer Graphics Forum, vol. 26, no. 2, pp. 214-226, June 2007.

[11] W. Wang, H. Pottmann, and Y. Liu, "Fitting B-spline curves to point clouds by curvature-based squared distance minimization," ACM Trans. Graph., vol. 25, no. 2, pp. 214-238, 2006.

[12] O. Chum, J. Matas, and J. Kittler, "Locally optimized ransac," in DAGM 2003: Proceedings of the 25th DAGM Symposium, ser. LNCS, J. v. L. G. Goos, J. Hartmanis, Ed., no. 2781. Heidelberger Platz 3, 14197, Berlin, Germany: Springer-Verlag, September 2003, pp. 236243. 